\title{
Clusters of PMS stars in the Magellanic Clouds: the case of LH95
}

\author{
Nicola Da Rio \\ Max Planck Institute for Astronomy, Heidelberg, Germany
}

\begin{abstract}
We present a study of the pre-main-sequence (PMS) population in the stellar association LH95 in the LMC, based on the deepest HST/ACS photometry ever taken in a region of the Magellanic Clouds. This association hosts $\sim 2500$ PMS stars, distributed in three main subclusters. We isolate this population by subtracting the LMC field, study the reddening distribution which peaks at $A_{V} \sim 0.5 \mathrm{mag}$, and assign masses, down to $0.2 \mathrm{M}_{\odot}$, to each member using a newly derived conversion of evolutionary models for the LMC metallicity and the ACS photometric system. We derive the first IMF in the subsolar regime ever measured outside our Galaxy, complete down to $0.4 \mathrm{M}_{\odot}$. It presents a flattening at $1 \mathrm{M}_{\odot}$, changing its slope from $x=2.05$ for intermediate-mass stars to $x=1.05$ in the subsolar regime. Correcting for unresolved binarity, it is compatible with the Galactic IMF. We do not find evidence of spatial variations of the IMF in the region. We study the ages of the PMS members using maximum-likelihood methods based on the location of the stars in the color-magnitude diagram with respect to $2 \mathrm{D}$ isochrones obtained by applying intrinsic and observational biases (differential extinction, variability, confusion, unresolved binarity) to modeled simple stellar populations. We find a most likely global age of $\sim 10 \mathrm{Myr}$, and demonstrate that neglecting the aforementioned biases leads to an underestimation of the cluster age of up to $50 \%$. We find that the observed luminosity spread is more than twice larger than can be explained by such biases, confirming that the star formation in LH95 lasted a few Myr.
\end{abstract}

Keywords. Magellanic Clouds, open clusters and associations: individual (LH95), stars: premain-sequence, stars: formation, stars: luminosity function, mass function

The full poster (in pdf format) is available at http://www.astro.iag.usp.br/〜iaus266/Posters/pDaRio.pdf. 